\title{
To What Extent Does It Pay to Approach Open Source Software for a Big Telco Player?
}

\author{
Massimo Banzi ${ }^{1}$, Guido Bruno ${ }^{2}$, and Giovanni Caire ${ }^{3}$ \\ ${ }^{1}$ Telecom Italia - Technology \& Operations, \\ Innovation Architecture and Quality dep., \\ Via Zambra 1, 38100 Trento Italy \\ Free Univerisity of Bolzano-Boozen Italy \\ massimo.banzi@telecomitalia.it \\ 2 Telecom Italia - Technology \& Operations, \\ Innovation Architecture and Quality dep., \\ Via Reiss Romoli 274, 10148 Torino Italy \\ guido.bruno@telecomitalia.it \\ 3 Telecom Italia - Technology \& Operations, \\ Innovation Architecture and Quality dep., \\ Via Reiss Romoli 274, 10148 Torino Italy \\ giovanni.caire@telecomitalia.it
}

\begin{abstract}
In this paper we describe the strategy under adoption in Telecom Italia (TI) Technology Department toward open source software. This stems from trying to create synergy among big Telco Player to increase knowledge and influence over strategic communities to the evaluation of the creation of new communities over internally developed applications. In particular here the approach and the expectations in starting the community on WADE (Workflow and Agent Development Environment) is described. This is a platform used to develop mission critical applications and is the main evolution of JADE a popular Open Source framework for the development of interoperable intelligent multi-agent systems. It adds to JADE the support for the execution of tasks defined according to the workflow metaphor as well as a number of mechanisms that help managing the complexity of the distribution both in terms of administration and fault tolerance. The idea is to use WADE as a mean to gather critical information on the opportunity of approaching OS as a strategic mean toward the development of always more important application in Operating Support System for TI, possibly also involving other great Telco Players For this reason great care is being paid in setting up the Community environment and in deciding which metrics are to be extracted from it, since the result will be the input for a strategic decision in TI.
\end{abstract}




\section{Introduction}

What is the value in OS for a Big Telco Player such as TelecomItalia?

With 7.3 million broadband connections (retail and wholesale) 0, Telecom Italia is currently the leading operator in the national TLC market. It has one of the most penetrating and advanced network in Europe, with an extension of over 107 million $\mathrm{Km}$ in copper lines (access) and 3.8 million $\mathrm{km}$ of optical fibers (transport and access).

This is a question that is intriguing OSS Software Factory Management in TI Technology Department. Nowadays the constant search for reduction of Total Cost of Ownership (TCO) is forcing more and more to find solutions effective, but suitable to fulfill all OSS processes in a modern big Telco Company (Service Delivery, Network Creation and Inventory, Workforce Management, etc.). The analysis toward Open Source Software ha been obviously anticipated by initiatives such as the Service Rationalization and the Application Rationalization, to reduce redundancy of services and tools, but the obvious consequence has been the thought whether we could substitute Commercial tools with OS ones without loosing confidence and efficiency.

The first approach toward OS has been driven by a set of indicators that have been formalized as follows 0 :

- The product has to fulfil a need and provide a well defined and understood service so that it can become a commodity.

- It ha to be a leader on the market in its context

- There should be a high number of "customers" that are also "contributors" for the product

- It has to be modular

- It must have a robust and well designed core

- The community needs to be large and talented.

This was the theory.

What actually happened was that, also according to several surveys 0 , the core of $\boldsymbol{u s e}$ of $O S$ in both mission-critical and non-mission-critical IT environments in TI rate higher for infrastructure than application software, and infrastructure software uses OS components in conjunction with proprietary software.

More, in TI the use of OS applications in OSS vertical solutions is almost absent.

The reason stands mostly in the opportunistic adoption of $O S$ in every single case, not driven by a real strategy.

In other words, in using OS software, an "opportunistic" approach (simply just use it) can be adopted or "a strategic one".

In the first case OS can be selected eventually also just as a proof of concept (using it simply as a leverage against COTS vendors). Or it can be simply used, directly, without even leaning on the community: it is downloaded, embedded in proprietary software and fully managed as an integral part of the solution.

Clearly in this case the community is used simply as a source of information for solving possible problems that can arise. No contribution is given. 
In the second case two situations can arise

- if a community and a suitable OS solution considered as important does exist, it is necessary

- the exploitation of community as a way to reduce development costs, accelerate the availability of new features, keep the product more robust trough the high number of users and contributors

- since the product is probably to become an integral part in the player products portfolio, strong Competence Centres participating the communities are a necessity to be able to influence selected strategic communities and the roadmap of the products.

- to keep a strong link between the innovation of the product in the community and the Telco production itself (basically an internal development branch has to be kept to validate every new added feature in the context the OS product is used by the company).

Clearly all these can be achieved only by actively participating the community, injecting it with resources and competences..

- If a community and a suitable OS solution does not exist, it is possible to evaluate the possibility of "open" software solution internally developed or to create a new community from scratch, joining several stakeholders needs.

Up to now TI just used the opportunistic approach. But now things are changing. The main reason are the arising consciousness that OS ensure best-of-breed solutions, improved ROI, and vendor independence and flexibility.

Moreover OS solutions reached a quality comparable with that of proprietary Software, and the possibility to lean upon third companies providing support on OS solutions thus reducing risk in OS adoption are removing the remaining doubts.

\section{Different approaches}

According to the above consideration, TI Technology department decided to approach OS with a real strategy, without also rejecting the possibility of an opportunistic approach for non strategic solutions.

\subsection{Synergy among big Telco players on existing communities}

As said, in case the community exist, the Player typically uses the OS product directly or mediated by a broker upon which it unloads part of the risks. 
Sometimes it also acquires from vendor or even brokers customized OS software. For whatever choice of OS adoption strategy, needs in OSS for most Telco operators are similar, and the competition among Big Players is on services not on software solutions.

So a question arose: why not to coordinate the interaction with OS communities?

This can result in several benefits, first of all the influence on the community can be stronger but also if brokers are used, these can be shared; contributions can be distributed as well as competences and the weight on board of the community can be stronger.

Finally a common action on the communities, on brokers, but also on vendors can result in the possibility of defining standards instead of undergoing them.

Hence the idea of proposing a working-table among Telco Players to promote and manage the creation of community specific Working Groups that homogeneously interact with the community, eventually enforcing and promoting single player initiatives on OSS.

\subsection{Creation of a new community}

If a suitable community does not exist, maybe it can result profitable to share an internally developed solution to extend the test base or also the development one if enough interest can be excited on the solution. This is the case of WADE where a solution based upon an existing Open Source platform (JADE 0) used to develop internal critical application has been chosen as a laboratory to verify the opportunity of such an approach.

The model that we decided to adopt is depicted in 0 . 


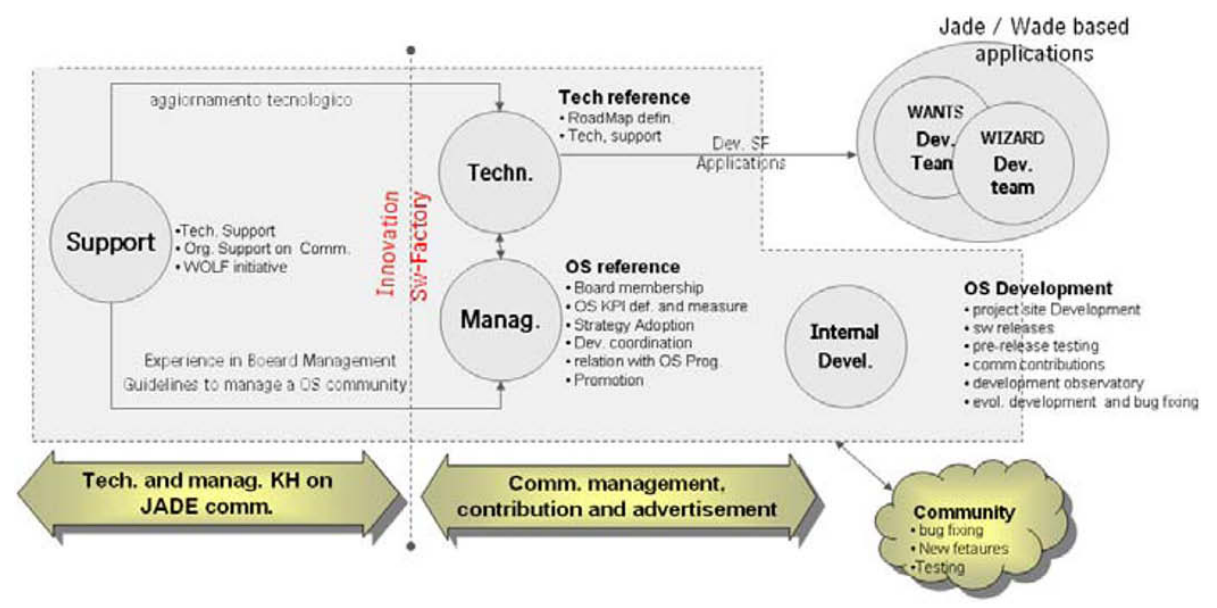

\section{Organizational scenario}

There, a Support Group has been identified, which is the original development team of WADE that clearly owns the major skill on the solution. It is engaged also in other activities and so it is expected to provide only a high level support to the initiative. A Technical manager for the specific solution has been identified within the Factory (the department in TI that actually develops or integrates software solutions). He currently is the responsible for all the applications developed on the top of WADE. He will also be responsible for deciding on the roadmap of the product within the arising community.

There is also a management role which is not just for the community created on WADE, but that is expected to manage all the solutions that will be opened. He will be responsible for the shared environment, the collection of metrics on it, its promotion, the coordination of the resources allocated on the community that will have to deal with internal builds, but also with releases, with the validation of community contributions, bug fixing, main evolution, etc.

The expectation is that we will be able to engage other companies in the initiative so that, others join the structure described in 0 . Another technical reference is expected to be identified in the "other" company to create a "WADE working group" as well as "other" developers to contribute and a Manager to be the only reference in the "other" company for the sharing of OS solutions between the two. The two managers will be also members of the Working-table described above. 


\subsection{Impacts}

What are the impacts on overall structure of the development and what the actions to be taken into consideration?

It is clear that in the creation of a working table a lot is to be done on the mentality. Apparently it is still rooted in many companies the idea that sharing collaboration with historical competitors can be a mistake. Give access to information and also the source code used to develop mission critical solution is a bad choice.

But the truth is that currently the standardization of OSS solutions causes few great vendors and consultants to share the market and provide different Telco Companies with the same solutions, eventually slightly customized.

What competitive advantage can be found in this? Better is consciously do the same thing but driving the choose, not being led individually to almost the same solution with multiplied costs.

What we are currently doing is the exploitation of already existing channels (TMF Operator Committee) to promote the initiative among big Telco Players and verify the interest on it.

In crating the new WADE community instead we have to face other problems. As said we want to be able to measure the impact that introducing OS can have either in the organization or in the quality of the software.

As clearly stated by 0 , opening up a project can add whole new sets of complexities, and cost more in the short term than simply keeping it in-house. Opening up means arranging the code to be comprehensible to complete strangers, so documentation needs to be clear where sometimes it is almost absent.

Moreover packaging is a topic that is underestimated when the development is internal, but in distributing it, it is necessary to create easy installable packages.

More if interest arise on the community, there is the added burden of answering developers' questions for a while before seeing any benefit from their presence.

This activity is much closer to promotion than to software development, but sometimes it is the first impact on the environment, on the care devoted to users, on the possibility of having support to ensure a success to the project, more than the embedded idea that can be successful.

Also management aspects are very different from those necessary in in-house development.

There are several developers, notoriously independent, who do not accept easily the idea that behind the product stands a big company. Even if the community will be done of just resources provided by different companies (and this is undoubtedly limiting) there is a coordinating activity far more complex than the one required for a homogenous group of co-located developers.

There should be a management conducting an activity closer to that of a psychologist that have to keep different people together giving them the consciousness that working together is more productive than working individually.

An Open Source community has to be treated more as a social network then as a developers team. This is also the reason of the importance of the development envi- 
ronment that must provide the easiest tools for communicating, sharing experiences and problems. In other words it has to be a place developers want to keep coming back to.

Finally legal aspect have to be carefully treated since one of the most diffused license for instance, the GNU Public License, as discussed in 0 , appears to have the ability to expose proprietary software developers to substantial risk if used improperly.

\subsection{Metrics}

The purpose of the initiative is that of "measuring" the opportunity of using OS as a resource for TI. This means that all possible measures, translated into "savings" has to be collected.

What saving means is clear, not as much how indirect saving can be collected and then monetized.

And in using OS there are not just savings, as repeatedly stated above, there are extra costs: the cost of setting up an environment to be accessed from outside, the cost of the restructuring the product to be open effectively. The cost of a parallel internal environment to reproduce the build before delivering the solution to internal projects, the cost of internal development and management, etc.

Clearly an expense has been activated to charge the effort to be devoted to such activities that are clearly identified, and this can rate the direct cost of the initiative.

Similarly we are collecting the effort spent in past years for maintaining WADE, for a comparison of the values.

But obviously we want also to measure the contribution given by the community, that cannot be measured in man/hours.

So the usual environmental metrics are collected 0 :

- The number and growth of "core" members divided by dominion

- The number and growth of downloads

- The number and growth of contributions either to the source code repository or to the mailing list (divided in developers and users, this measures the "vitality" of the community )

- The mean time to respond to an issue (again full and cleaned by our direct contributions)

- The fixing rate (internal, but more important form external contributors)

- The main fixing time from external contributors ("Reaction Rate")

As well as quality metrics:

- Delta times to manage the introduction of new features with respect to insourcing criteria

- Debugging times and quality

- Cohesion - change in cohesion - structural 0 
- Bugs (normalized on LOC, bug fixing mean time) mainly on critical components (the more changed ones)

Also organizational metrics are to be collected:

- Measure the level of control and influence on the community: how many internally proposed new features have been accepted by the community and implemented by others than internal contributors

Clearly all these need to be translated into a real value for Telecom Italia and so apart form translating the indicators into objective numbers, it is necessary to find conversion parameters to turn these into saved effort.

We are still working on all this.

\section{Conclusions}

As widely described TI is trying the approach to OS as a possible way for further reduce the TCO of its OSS.

The strategic relevance of WADE and its choose as trial for such an initiative demonstrates the interest of TI to approach Open Source as a real resource for its business.

The process has just started: a plan for the initiative is ready and starting from March 2008 WADE will have its own environment, mailing list, forum, etc. and a web site from which it will be downloadable.

A document describing the full set of metrics that will be collected is under development in collaboration with the University of Milano and these metrics will be systematically collected to measure the effectiveness of the initiative and to refine the environment according to result as well as on feedback from the community.

Every three months these metrics will be published to the management who has great interest in the initiative.

Concurrently ha thread with TMF has been started with the goal of starting a catalyst project on OS to give the initiative all the strength of an organization that has also tha aim of proposing standards within Telco community.

It's a bet we expect to win.

\section{References}

The individual shareholder Guide Oct. 2007 http://ticlub.telecomitalia.com/pdf/Guida_1H 2007_ottobre_EN.pdf

Solari A. , Le opportunità del modello Open Source; un percorso in Telecom Italia - Technical Information Services verso il SW Open Source, OSBAIT Nov. 2006

Laurie Wurster, User Survey Analysis: Open-Source Software Summits, North America and Europe, 2006, Gartner 
Rishab Aiyer Ghosh, Study on the:Economic impact of open source software on innovation and the competitiveness of the Information and Communication Technologies(ICT) sector in the EU, MERIT 2006,

Karl Fogel, Producing Open Source Software How to Run a Successful Free Software Project, http://producingoss.com/

JADE - Java Agent Development framework. http://jade.tilab.com.

Frost J., Some Economic \& Legal Aspects of Open Source Software, 2005, http://students. washington.edu/jjfrost/

Ardagna C., Damiani E., Frati F. FOCSE an OWA-based Evaluation Framework for OS adoption in Critical Environment, in Open Source Development, Adoption and Innovation, Springer 2007

Misic V., Measuring the Coherence of Software Product Line Architectures, Tech. report TR 06/03 - Univ. of Manitoba 